\title{
Análisis de la prima de riego en las mujeres auditoras: el caso español
}

\section{Analysis of the Risk Premium for Auditor Women: The Spanish Case}

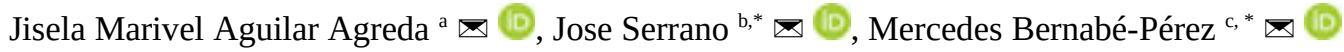 \\ $a, b, c)$ Facultad de Economía y Empresa. Universidad de Murcia, Spain
}

* Corresponding author: bernabe@um.es (Mercedes Bernabé-Pérez)

\section{Resumen}

Este estudio investiga la aplicación, por parte de las socias auditoras, de una prima de riesgo en las auditoras españolas de pequeñas y medianas empresas como herramienta de cobertura. Mediante técnicas econométricas se ha realizado un análisis univariante y multivariante a 2.536 observaciones de clientes auditados por veintiuna firmas de auditoría de pequeña y media dimensión durante el periodo 2002 y 2014 . Los resultados sugieren que las empresas auditadas pagan unas tarifas de auditoría más altas cuando el informe de auditoría es firmado por una mujer. La evidencia combinada en este estudio sugiere la existencia de una prima de riesgo femenina. Esta prima en los honorarios puede existir por las diferencias de género existentes a la hora de percibir y tolerar el riesgo. A diferencia de estudios anteriores se controla el esfuerzo del auditor a través de las horas dedicadas en cada uno de los trabajos, centrando el análisis en el segmento de pequeñas y medianas firmas de auditoría.

Palabras clave: género; riesgo; prima; esfuerzo; pequeños auditores

Clasificación JEL: J16; M42

\section{Abstract}

This study investigates the application by auditor women of a risk premium in Spanish audit firms of SME companies as a hedging tool. Using econometric models, it has been developed univariate and multivariate analysis over 2,536 observations of clients audited by twenty one small and medium-sized audit firms for the period of 2002 to 2012. In short, the results suggest that the audited companies pay higher tariffs audit when the audit report is signed by a woman. The combined evidence in this study suggests the existence of a female risk premium. This premium in fees may exist due to gender differences existing when perceiving and tolerating risk. Unlike previous studies, the audit effort has been controlled by the hours dedicated to each of the works and focused the analysis on the segment of SME audit firms.

Keywords: gender; risk; premium; effort; small auditing firms

JEL Classification: J16; M42 


\section{Introducción}

Los socios poseen un alto grado de autonomía para ejercer el juicio profesional en el curso de un contrato (Knechel, Niemi y Zerni, 2013). Esta autonomía también comprende el poder de influir en las horas establecidas de auditoría, así como los precios en cada encargo. En el actual escenario de competencia, el auditor debe de minimizar los riesgos de auditoría y maximizar los honorarios y, en función de la elección individual del auditor firmante, podrá optar por una de las distintas herramientas de cobertura de riesgos, entre ellas, la prima de riesgo en honorarios.

El objetivo del presente estudio es investigar si las mujeres auditoras españolas, socias de pequeñas y medianas firmas, tienen en cuenta el riesgo de negocio que se puede presentar, y por tanto, si en la fijación de precios de cada uno de los contratos que mantienen con sus clientes incluyen una prima de riesgo, para hacer frente a las posibles pérdidas futuras a las que se pueden enfrentar.

Si los estados financieros auditados contienen algún error que no se ha detectado en el proceso, el auditor se enfrentará a una posible pérdida de reputación, así como a posibles litigios y sanciones por parte del Instituto de Contabilidad y Auditoría de Cuentas (ICAC), que vienen a poner de manifiesto el riesgo de negocio. Para hacer frente a este el riesgo, el auditor puede valerse de distintas herramientas como son, entre otras, la gestión de cartera de clientes, la emisión de una opinión modificada o la incorporación en el precio de una prima de riesgo. El presente trabajo se centra en la prima de riesgo como herramienta de cobertura, peroal contrario que en estudios anteriores, se controla el esfuerzo del auditor a través de las horas dedicadas en cada uno de los trabajos, centrando el análisis en las socias firmantes de pequeñas y medianas firmas de auditoría.

La existencia de varios estudios no relacionados con el mundo de la contabilidad, donde existen elevadas diferencias de género, principalmenteen la asunción de riesgos (Byrnes, Miller, y Schafer, 1999), lleva a pensar que pueden existir diferencias significativas en la aplicación de primas de riesgo por parte de las mujeres auditoras. En este sentido, la aplicación de una prima de riesgo no deja de ser un reflejo de la capacidad de la auditora de identificar riesgos, lo que evidencia un nivel de calidad del trabajo. Este estudio contribuye a la literatura limitada pero creciente sobre la calidad de auditoría en función de si esta es realizada por una mujer o por un hombre (por ejemplo, Gold, Hunton y Gomaa, 2009; Hardies, Breesch y Branson, 2014; Ittonen, Peni y Vähämaa, 2013).

Concretamente, se amplía el estudio de Ittonen y Peni (2012) en el que se obtuvo que las firmas con mujeres auditoras tienen honorarios más altos que las empresas con auditores hombres, para una muestra formada por empresas de Finlandia, Dinamarca y Suecia, así como el estudio de Hardies, Breesch y Branson (2015), compuesto por empresas belgas. Sin embargo, el que varíen las brechas salariales por género entre los distintos países de Europa y el que este estudio se centre en el segmento de las pequeñas y medianas firmas de auditoría, que tienen una mayor competencia vía precios, hace no poder concluir a priori que se produzcan los mismos resultados en el mercado español. Además, se usa un conjunto de datos que ha permitido diseñar una investigación de mayor calidad ya que se utiliza como subrogado del esfuerzo del auditor, las horas declaradas al ICAC, eliminando la posibilidad de que la prima de riesgo refleje un incremento adicional del trabajo realizado.

En base a la revisión bibliográfica, este estudio sería el primero que analiza el impacto del género del auditor firmante en las primas de riesgo aplicadas en pequeñas y medianas firmas auditoras dentro del mercado español. El trabajo está organizado de la siguiente manera: la siguiente sección contiene la revisión de la literatura existente sobre la prima de riesgo, género y el planteamiento de la hipótesis. En la tercera sección, se describe el método de investigación y recopilación de datos. En la cuarta sección se exponen los resultados. Finalmente, se concluye 
con una discusión general de los resultados, las limitaciones de esta investigación y las posibles investigaciones futuras.

\section{Revisión bibliográfica y determinación de la hipótesis}

La actividad de auditoría de cuentas se caracteriza principalmente por la importancia pública que desempeña al prestar un servicio a la entidad revisada y afectar e interesar no sólo a ésta, sino también a los terceros que mantengan o puedan mantener relaciones con la misma y cuyas decisiones se basen en los estados financieros auditados. El que un tercero base susingresos futuros en la opinión del auditor hace que el primero ponga en el segundo una serie de expectativas que no siempre tienen por qué darse por satisfechas. En caso de que no lo sean, puede ocasionar futuras pérdidas para el auditor por demandas, pérdidas de reputación o incluso sanciones.

Aun habiendo alcanzado el nivel de calidad exigido por las Normas Internacionales de Auditoría (NIA), el auditor puede no haber satisfecho las expectativas de terceros, siendo difícil un incremento de horas de trabajo, y considerando optar por incorporar un incremento en los precios para cubrir la posible pérdida futura (Pratt y Stice, 1994; Simunic y Stein, 1996; Houston, Petters y Prat, 1999).

La norma internacional de auditoría (NIA-ES) 200 es la que establece que el auditor debe obtener una "evidencia de auditoría suficiente y adecuada para reducir el riesgo de auditoría (es decir, el riesgo de que el auditor exprese una opinión inadecuada cuando los estados financieros contengan incorrecciones materiales) a un nivel aceptablemente bajo" (BOICAC, 2015, p. 2).

No obstante, una seguridad razonable no significa un grado absoluto de seguridad, debidoa que existen limitacionesinherentesa la auditoría que hacen que la mayor parte de la evidencia obtenida, a partir de la cual el auditor alcanza conclusiones y en la que basa su opinión, sea más convincente que concluyente. Estas limitaciones puede ser la propia situación financiera del cliente (Niemi, 2004), la regulación del sector en el que opera el auditado o el riesgo inherente no eliminado.

El que las herramientas de cobertura de riesgos a disposición del auditor únicamente otorguen una seguridad razonable y no absoluta y la posibilidad de que un eventual default del cliente auditado genere pérdidas en terceros, son el origen de que el auditor esté sometido a un riesgo de negocio (Stice, 1991; Carcello y Palmrose, 1994; Lys y Watts, 1994; ZhanShu, 2000), definido como el riesgo de que el auditor incurra en pérdidas con motivo de su relación con el cliente auditado.

Para hacer frente a los riesgos sobre continuidad a nivel internacional se diseñó la ISO 22301 sobre Sistemas de Gestión de Continuidad de Negocio, consistente en identificar las posibles amenazas y orientar sus capacidades con el fin de preparar a la organización para una respuesta que minimizara el daño ocasional que puede suponer el acaecimiento de hechos que pongan en peligro la continuidad de la firma. En este trabajo no se tiene constancia de la implantación formal de la ISO 22301 en el sector de la auditoría de cuentas, si bien, el auditor utiliza distintas herramientas como son la gestión de cartera de clientes, la emisión de una opinión modificada o la incorporación en el precio de una prima de riesgo.

El poder de elección de las distintas herramientas de cobertura frente al riesgo reside en el auditor firmante del encargo, reforzando esta idea las NIAs al otorgar al juicio del auditor la capacidad de fijar las herramientas de cobertura como materialidad (esfuerzo del auditor), honorarios (prima de riegos) o continuidad del cliente. Parece claro que cualquier rasgo distintivo entre un auditor y otro puede implicar el uso de distintas herramientas de cobertura. 
Las diferencias entre individuos a la hora de adoptar decisiones han sido ampliamente estudiadas en distintos ámbitos de la ciencia (por ejemplo, Byrnes, Miller, y Schafer, 1999). Esto conduce al planteamiento de si las posibles diferencias entre mujeres y hombres auditores pueden suponer distintos juicios que impliquen la elección de distintas estrategias antes el riesgo, si bien este estudio se centra exclusivamente en la incorporación de una prima en los honorarios.

A nivel internacional, dentro del ámbito de la auditoría se puede encontrar un número cada vez mayor de estudios que se centran en las diferencias entre mujeres y hombres auditores (por ejemplo, Gold et al., 2009; Hardies et al., 2014; Ittonen et al., 2013) con resultados similares a los que se centran en el campo de la contabilidad y las finanzas (Sundén y Surette, 1998; Jianakoplos y Bernasek, 1998; Olsen y Cox, 2001; Graham, Stendardi, Myers y Graham, 2002; Dwyer, Gilkeson y List, 2002; Watson y Robinson, 2003; Watson y McNaughton, 2007). Las mujeres tienden a ser más conservadoras, concluyendo un pequeño número de autores que tales diferencias pueden afectar los juicios del auditor, que es, en definitiva, asunción de riesgos (Chung y Monroe 2001; Gold et al. 2009).

Ittonen y Peni (2012) fueron pioneros en la investigación sobre la existencia de una prima de riesgo femenina. Llevaron a cabo un estudio sobre una muestra de 715 observaciones. Como principales resultados encontraron que las empresas (en Finlandia, Dinamarca y Suecia) con socias auditoras tenían unas mayores tarifas que las empresas con socios auditores. Sin embargo, no está claro en qué medida los resultados de este estudio pueden generalizarse a otros ámbitos.

Posteriormente, Hardies, Breesch y Branson (2015) amplían el estudio exploratorio de Ittonen y Peni (2012). Dicho estudio proporciona evidencia de la existencia de una prima de riesgo femenina en un contexto nacional compuesto por empresas belgas y, además, en él se empleó un gran número de datos que les permitió diseñar una investigación de mayor calidad, eliminando así las posibilidades de que la existencia de prima de riesgoreflejara otras diferencias entre ambos sexos (por ejemplo, la especialización) o diferencias en las características de sus clientes (tamaño de la empresa auditada) que pudieran afectar a los honorarios. No obstante, mencionan que no se puede descartar por completo la posibilidad de que no se hayan controlado los factores que podrían afectar la relación entre los honorarios y el género del auditor.

\subsection{Hipótesis a contrastar}

Como se ha mencionado anteriormente, varios estudios han intentado demostrar empíricamente la existencia de una aversión al riesgo distinta entre hombres y mujeres, en concreto, a la hora de establecer una prima de riesgo en los honorarios. Sin embargo, dichos resultados no son del todo concluyentes, pues no controlan de forma efectiva el esfuerzo del auditor, varían en función del país y se centran en sociedades auditadas cotizadas o en las grandes firmas auditoras, que tienen una mayor visibilidad que el segmento de las pequeñas y medianas firmas. Por ende, la pretensión de este estudio es aportar evidencia sobre un mercado de auditoría de pequeñas y medianas empresas del que no se han realizado trabajos sobre la política de precios en relación con la existencia de una prima de riesgo mayor en las socias auditoras, teniendo en cuenta el esfuerzo del auditor.

Cabe destacar que la existencia de una fuerte competencia en el mercado de pequeñas y medianas firmas hace que los auditores reduzcan sus precios por debajo del coste marginal de producción del servicio, con el objetivo de mantener o capturar nuevos clientes. Para determinar si realmente las auditoras de pequeñas y medianas firmas en España incorporan a sus clientes una mayor prima de negocio en sus honorarios, se plantea la siguiente hipótesis en términos nulos:

H1: Las auditoras no cargan una mayor prima de emisión a los clientes que los auditores para cubrirse del riesgo de negocio. 


\section{Estudio empírico}

\subsection{Muestra}

Para poder llevar a cabo este estudio se ha seleccionado aleatoriamente un subconjunto de la población, representativo del segmento de medianas y pequeñas firmas de auditoría. Entre un total de 1.384 firmas inscritas en el Registro Oficial de Auditores de Cuentas (ROAC), una vez descartadas por su gran tamaño las Big Four y las denominadas Second-tie y siguiendo criterios de dispersión geográfica, se solicitó a 21 firmas losmodelos03presentadosentreel período 2002-2014, obteniendo respuestas de 11 de ellas. Dichos datos se han cruzado con la base de datos SABI para completar las distintas variables sujetas a investigación. En total, se han analizado 2.536 observaciones de clientes auditados por auditores de pequeña y mediana dimensión. Del total de informes, 206 fueron emitidos por socias y 2.330 por socios auditores.

Según el artículo 24.2 de la Ley de Auditoría, las empresas auditoras deberán comunicar al ICAC anualmente en el modelo 03 las horas y honorarios facturados a cada entidad auditada, distinguiendo las que corresponden a servicios de auditoría de cuentas y otros servicios, así como cualquier otra información que precise el ICAC para el ejercicio de sus funciones. Dicho modelo incluye el CIF/ID y denominación social de la entidad social, si el informe corresponde a la empresa individual o al consolidado, fecha de cierre del primer ejercicio auditado, información sobre si el ejercicio auditado es el de constitución, transformación o fusión, fecha de cierre del ejercicio auditado, tipo deentidad (entidades de crédito, aseguradoras, etc.), el importe de la cifra del activo del ejercicio auditado y anterior, el importe netodela cifra de negocios, el número medio de trabajadores, tipo de trabajo (auditoría de las cuentas anuales o de otros estados financieros, así como el carácter obligatorio o voluntario), fecha de emisión del informe, autor firmante, coauditoría, tipo de trabajo, provincia, honorarios y facturación a la entidad auditada por trabajos distintos a la auditoría.

\subsection{Definición del modelo}

Para determinar si la prima de riesgo es mayor cuando es mujer la socia firmante,seestimael siguiente modelo de precios basado en el inicial de Simunic (1980) y desarrollado posteriormente en estudios de género por Ittonen y Peni (2012) y Hardies et al. (2015):

$$
\begin{array}{r}
\text { HONORARIOS }_{i t}=\beta_{0}+\beta_{1} L E V_{i t}+\beta_{2} C A T A_{i t}+\beta_{3} \text { ROA }_{i t}+\beta_{4} P Y \text { LOS }_{i t}+ \\
\beta_{5} \text { OPINION }_{i t}+\beta_{6} \text { NUEVO }_{i t}+\beta_{7} \text { SEX }_{i t}+\beta_{8} \text { HORAS }_{i t}+\beta_{9} \text { OTROSTRABAOS ROS }_{i t}+ \\
\beta_{10} \text { CITY }_{i t}+\beta_{11} \sum_{j=1}^{4} S E C T O R_{j}+\beta_{12} \sum_{k=2002}^{2014} A N O_{k}+\varepsilon
\end{array}
$$

La variable dependiente "HONORARIOS", representa la remuneración recibidapor el servicio de auditoría, del cual se ha tomado el logaritmo neperiano, para evitar problemas de escala.

Para la existencia de riesgo de negocio del auditor es condición necesaria que el usuario de la información incurra en pérdidas, y ello se puede originar por un default del cliente auditado o por una baja rentabilidad de éste.

Para medir el riesgo de negocio se utilizan diversas variables relacionadas con la situación financiera del cliente. En primer lugar, la variable LEV (DeFond, Jere y Wong, 2000) determina el apalancamiento financiero de la empresa, que se obtiene dividiendo las deudas financieras a largo plazo entre el total de activos. La relación esperada entre la variable y los honorarios es positiva, ya que cuanto mayor sea el riesgo financiero del cliente, el riesgo de quiebra será mayor y se aplicarán unos honorarios más elevados.

Se utiliza la variable CATA, que se calcula dividiendo el activo circulante entre el total de activo (Francis y Simons, 1987), para evaluar la solvencia del cliente a corto plazo. La existencia en términos relativos de un activo más líquido puede ser interpretada por parte del auditor como 
una mejor solvencia a corto plazo, disminuyendo así el riesgo de negocio, esperando una relación negativa entre esta variable y la variable dependiente, ya que mientras más elevada sea la variable menor es el riesgo de negocio.

Desde el punto de vista del impacto de la rentabilidad en el riesgo de negocio, se ha analizado tanto el resultado del ejercicio auditado como el del año anterior. La variable ROA se obtiene dividiendo el beneficio de la actividad entre el total de activos, esperando que su relación sea negativa y PYLOSSque toma el valor 1 cuando en el año anterior la empresa sufrió pérdidas y 0 en el caso contrario (DeFond et al., 2000 y Hardies et al., 2015). Se espera una relación positiva entre la variable dependiente y PYLOSS debido a que la existencia de pérdidas incrementa el riesgo financiero del cliente.

Otra variable que varios autores vinculan con el riesgo de negocio (Francis y Simon, 1987 y Simunic, 1980) es la OPINION, que toma el valor 1 si el informe que se emite posee alguna salvedad y 0 cuando dicho informe es favorable. En este sentido, se ha venido argumentado que al emitir el auditor un informe modificado se protege frente a las posibles demandas, reduciendo así el riesgo de negocio. La variable NUEVO es una variable dicotómica que toma el valor 1 cuando el cliente es nuevo y 0 en caso contrario. Se espera una relación positiva respeto a la variable honorarios. Diversos autores como Bell, Doogar y Solomon (2008), han señalado que un cliente nuevo generará al auditor un mayor riesgo debido a la falta de conocimiento sobre el mismo.

La variable SEX es dicotómica y toma el valor 1 si el auditor es mujer y 0 en el caso contrario. Se espera un coeficiente positivo entre esta variable y los honorarios, pues como se ha desarrollado anteriormente, las mujeres son más adversas al riesgo, lo que les llevaría a incluir una prima de riesgo en caso de que detectasen la existencia de riesgo de negocio.

Las variables de control usadas son aquellas que informan sobre los recursos asignados en cada encargo por el auditor, así como el sector en el que se encuentra cada empresa auditada y el año en el que se ha realizado el servicio. En cuanto a los recursos asignados por auditora a cada trabajo, la variable HORAS nos refleja el esfuerzo de auditoría en cada contrato, calculada en logaritmos neperianos (Bell, Landsman y Shackelford, 2001; Jhonstone y Bedard, 2003). Se espera una relación positiva de esta variable con los honorarios, ya que los honorarios serán mayores cuanto mayor sea el tiempo empleado en el encargo. Y la variable OTROSTRABAJOS incorpora aquellos servicios distintos al servicio de auditoría, para así medir la posible influencia de economías de escala en caso de que la empresa auditora preste otros servicios (por ejemplo, Monterrey y Sánchez, 2007).

Para controlar la influencia del coste de la vida en los honorarios, la variable CITY toma el valor 1 si la firma tiene sede en Madrid o Barcelona y 0 en caso contrario. De acuerdo con Chan, Ezzamel y William (1993), Brinn, Peel y Roberts (1994), O'Sullivan (2000) y Beattie, Goodacre, Pratt y Stevenson (2001), en las grandes ciudades los precios cargados a sus clientes son mayores en comparación con los precios que se fijan en pequeñas ciudades.

Además, la variable SECTOR actúa como variable de control porque varios autores (Simunic, 1980; Palmrose,1986) han demostrado que existe una relación entreel riesgo de negocio del auditor y el sector de actividad. Por ello, se considera el efecto que puede tener y se agrupan las empresas auditadas según la Clasificación Nacional de Actividades Económicas (CNAE), bien en los sectores de la agricultura, industria, construcción, suministros y actividades inmobiliarias. Dicha variable es dicotómica tomando el valor 1 si se encuentra la empresa auditada en algunos de los sectores y 0 en caso contrario. Por otro lado, se incorpora la variable AÑO para controlar el efecto temporal delos datos. Es dicotómica tomando el valor 1 si se encuentra en el periodo correspondiente y 0 en caso contrario.

En la (Tabla 1) se observan todas las variables incluidas en el modelo, así como su tipo (categórica o métrica), su medida y el signo esperado de las mismas, excepto las variables SECTOR y AÑO. "Honorarios" es la variable dependiente y el resto de variables son las explicativas o independientes. 


\begin{tabular}{|c|c|c|c|c|c|c|}
\hline \multicolumn{7}{|c|}{ Tabla 1. Variables del modelo } \\
\hline VARIABLE & TIPO & \multicolumn{4}{|c|}{ MEDIDA } & $\begin{array}{l}\text { Signo } \\
\text { esperado }\end{array}$ \\
\hline $\begin{array}{l}\text { HONORARIOS } \\
\text { (Variable dependiente) }\end{array}$ & Métrica & \multicolumn{4}{|c|}{ Logaritmo neperiano de los ingresos por cliente. } & --- \\
\hline LEV & Métrica & \multicolumn{4}{|c|}{ Deudas a largo plazo/Total Activo } & Positiva \\
\hline CATA & Métrica & \multicolumn{4}{|c|}{ Activo circulante/Total Activo } & Negativa \\
\hline ROA & Métrica & \multicolumn{4}{|c|}{ Beneficio de actividades ordinarias/Total Activo } & Negativa \\
\hline PYLOSS & Métrica & \multicolumn{4}{|c|}{$\begin{array}{l}1 \text { cuando la empresa tiene pérdidas en el año } \\
\text { anterior y } 0 \text { en caso contrario }\end{array}$} & Positiva \\
\hline OPINION & Dicotómica & \multicolumn{4}{|c|}{$\begin{array}{l}1 \text { si el informe posee alguna salvedad y } 0 \text { cuando } \\
\text { es favorable }\end{array}$} & ---- \\
\hline NUEVO & Dicotómica & \multicolumn{4}{|c|}{1 cuando el cliente es nuevo y 0 en caso contrario } & Positiva \\
\hline SEX & Dicotómica & \multicolumn{4}{|c|}{1 si el auditor es mujer y 0 en el caso contrario } & Positiva \\
\hline HORAS & Métrica & \multicolumn{4}{|c|}{$\begin{array}{l}\text { Logaritmo neperiano de las horas declaradas por } \\
\text { los auditores en cada encargo a la auditoría }\end{array}$} & Positiva \\
\hline OTROSTRABAJOS & Métrica & \multicolumn{4}{|c|}{$\begin{array}{l}\text { Logaritmo neperiano de los ingresos percibidos por } \\
\text { servicios distintos a la auditoría }\end{array}$} & Positiva \\
\hline CITY & Dicotómica & \multicolumn{4}{|c|}{1 si la firma tiene sede en Madrid o Barcelona } & Positiva \\
\hline \multicolumn{7}{|c|}{ Fuente: elaboración propia. } \\
\hline \multicolumn{7}{|c|}{ Tabla 2. Estadísticos descriptivos } \\
\hline \multicolumn{3}{|c|}{ Media } & Desviación típica & Mínimo & Máximo & \\
\hline \multicolumn{3}{|c|}{0,12} & 0,14 & 0,00 & 0,88 & \\
\hline CATA & \multicolumn{2}{|c|}{0,63} & 18,19 & 0,00 & 1 & \\
\hline ROA & \multicolumn{2}{|c|}{0,12} & 1,70 & $-1,88$ & 58,55 & \\
\hline PYLOSS & \multicolumn{2}{|c|}{0,08} & - & 0 & 1 & \\
\hline OPINION & \multicolumn{2}{|c|}{0,23} & & 0 & 1 & \\
\hline NUEVO & & 0,13 & - & 0 & 1 & \\
\hline SEX & & 0,08 & - & 0 & 1 & \\
\hline OTROSTRAE & AJOS & 0,11 & - & 0 & 1 & \\
\hline CITY & & 0,27 & - & 0 & 1 & \\
\hline
\end{tabular}

\section{Resultados empíricos}

En la (Tabla 2) se recogen los estadísticos descriptivos usualmente utilizados en la literatura para describir los datos muestrales. Los datos que aparecen en la (Tabla 2) revelan que las empresas de la muestra gozan de un buen comportamiento en relación con su solvencia (CATA) y endeudamiento; si se atiende a la variable LEV, son empresas con poca deuda de carácter financiero, aspecto que refleja la dificultad de acceso al crédito durante el periodo de crisis financiera. La rentabilidad media se sitúa en un $12 \%$, por lo que puede inferirse que no existen problemas respecto a este extremo. Este dato se ve corroborado por la variable PYLOSS que revela que sólo aproximadamente un $8 \%$ de empresas incurrieron en pérdidas en el ejercicio anterior. Igualmente se refleja que aproximadamente un $23 \%$ de las opiniones emitidas por los auditores respecto a las empresas de la muestra recogen alguna salvedad.

Aunque no se cuenta con cifras sobre el porcentaje de informes cualificados emitidos para el total de empresas auditadas en el mercado de auditoría español, la Comisión Nacional del 
Mercado de Valores (CNMV) sí publica estos datos para las compañías cotizadas, siendo este porcentaje del 2,3\% para informes emitidos sobre cuentas anuales del ejercicio 2016 (véase Expansión, 16 de febrero de 2018). Por tanto, si se atiende únicamente a esta variable, se podría inferir que las empresas de esta muestra generan mayor riesgo de negocio a los auditores que los clientes tipo de las firmas multinacionales. También cabe resaltar queaproximadamente un $13 \%$ de los clientes son nuevos, y que entorno al $27 \%$ de las empresas han sido auditadas por firmas con sede en las grandes capitales de la península: Barcelona y/o Madrid.

En relación con los honorarios y las horas de trabajo de cada encargo, los valores de la muestra aparecen reflejados en la (Tabla 3). En el panel A de dicha tabla se recogen los estadísticos descriptivos para los honorarios y las horas de cada encargo. En el panel B aparecen estas mismas variables descritas por los distintos períodos objeto del estudio con el fin de analizar la evolución temporal que alcanzan. Y en el panel C se recoge la distribución sectorial de los honorarios y horas.

Por último, hay que reseñar que únicamente el $8 \%$ de los informes que componen esta muestra fueron emitidos por mujeres. El porcentaje es sensiblemente inferior al que se refleja en el Informe sobre la situación de la auditoría en España (2016) realizado por el Instituto de Censores Jurados de Cuentas de España (ICJCE) para los ejercicios 2006-2016, que sitúa el porcentaje de socias de auditoría en un $21 \%$. En dicho informe no se distinguen entre las grandes y pequeñas firmas, por lo que se puede entender que debido a la visibilidad de las Big-Four, tengan mayor incentivo reputacional para facilitar la incorporación a la mujer en la dirección de los despachos.

Según los resultados reflejados en el Panel A de la (Tabla 3), se observa que los honorarios medios cargados por los auditores de la muestra se sitúan en 6.187,20 euros. Si se analiza la evolución temporal de estos honorarios a lo largo del período objeto de estudio (Panel B, Tabla 3), se puede apreciar de forma casi general un incremento de los mismos, hasta los ejercicios 2011 y 2012 que suponen un punto de inflexión, descendiendo paulatinamente en los años siguientes, fruto probablemente del descenso de clientes y del incremento de la competencia vía precios, si bien la diferencia entre el último y el primer período de este estudio, de 2.264,01 euros, representa un incremento de aproximadamente el 53,87\%.

Igualmente se puede precisar que existen diferencias apreciables en los honorarios cargados entre los distintos sectores de actividad, lo cual podría venir explicado bien por la

Tabla 3: Estadísticos descriptivos honorarios y horas por encargo

\begin{tabular}{|c|c|c|c|c|c|c|c|}
\hline \multicolumn{8}{|c|}{ Panel A: Estadísticos descriptivos para los honorarios y las horas de cada encargo } \\
\hline Variable & \multicolumn{2}{|r|}{ Media } & \multicolumn{2}{|c|}{ Desviación } & Mínimo & \multicolumn{2}{|r|}{ Máximo } \\
\hline Honorarios & \multicolumn{2}{|r|}{$6.187,04$} & \multicolumn{2}{|c|}{$2.965,71$} & 600 & \multicolumn{2}{|r|}{30.000} \\
\hline Horas & \multicolumn{2}{|r|}{122,46} & \multicolumn{2}{|c|}{55,81} & 11 & \multicolumn{2}{|r|}{470,00} \\
\hline \multicolumn{8}{|c|}{ Panel B: Estadísticos descriptivos por periodo } \\
\hline Variable & & 2002 & 2003 & 2004 & 2005 & 2006 & 2007 \\
\hline Honorarios & & $4.202,61$ & $4.613,31$ & $4.985,79$ & $5.328,51$ & $5.395,99$ & $6.036,05$ \\
\hline Horas & & 142,25 & 139,47 & 141,52 & 137,56 & 127,59 & 130,84 \\
\hline Variable & 2008 & 2009 & 2010 & 2011 & 2012 & 2013 & 2014 \\
\hline Honorarios & $6.478,42$ & $6.391,19$ & $6.277,23$ & $6.634,65$ & $6.680,44$ & $6.568,37$ & $6.466,62$ \\
\hline Horas & 127,79 & 123,18 & 118,16 & 117,50 & 114,46 & 112,17 & 111,72 \\
\hline \multicolumn{8}{|c|}{ Panel C: Distribución sectorial de horas y honorarios } \\
\hline Variable & \multicolumn{2}{|c|}{ Agricultura } & Industria & Construc & \multicolumn{2}{|c|}{ Suministros } & $\begin{array}{l}\text { Actividades } \\
\text { inmobiliarias }\end{array}$ \\
\hline $\begin{array}{c}\text { Honorarios } \\
\text { Horas }\end{array}$ & \multicolumn{2}{|c|}{$\begin{array}{c}6.124,20 \\
114,50\end{array}$} & $\begin{array}{c}6.657,37 \\
135,71\end{array}$ & $\begin{array}{r}6.325,7 \\
128,97\end{array}$ & \multicolumn{2}{|c|}{$\begin{array}{c}6.400,14 \\
118,39\end{array}$} & $\begin{array}{c}4.088,47 \\
68,30\end{array}$ \\
\hline
\end{tabular}

Fuente: elaboración propia. 
desigual dificultad de realizar las auditorías en los distintos sectores de actividad, bien por el diferente riesgo de negocio que los distintos sectores pueden generar (Panel C, Tabla 3). De esta forma, puede destacarse que los honorarios son mayores en el sector industria, mientras que en el sector de actividades inmobiliarias el importe de los honorarios es menor; por otro lado, fue este último el sector que más se resintió durante el periodo de crisis, por lo que se puede percibir, otra vez, la competencia vía precios que existe en este segmento del mercado. Respecto a la variable horas, que como ya se ha destacado anteriormente refleja el esfuerzo realizado por los auditores en la ejecución del encargo, su valor medio es de 122,46 horas (Panel A, Tabla 3). A diferencia de los honorarios totales, se puede observar (Panel B, Tabla 3) una reducción paulatina del esfuerzo a lo largo del período objeto del estudio, lo cual puede resultar consistente con la tesis de la existencia de economías de aprendizaje y la aplicación de nuevas tecnologías al proceso. La diferencia entre el último y el primer ejercicio de este estudio es de 30,53 horas, lo que representa un porcentaje de reducción del esfuerzo de auditoría en un 27,32\%.

En la (Tabla 4) se presenta un análisis univariante de comparación de medias para analizar si las variables del modelo se comportan de forma distinta en dos submuestras. Para determinar ambas sub-muestras se ha dividido la muestra en función del sexo del auditor firmante: mujer $u$ hombre. Para analizar si existen diferencias significativas entre ambas sub-muestras se ha utilizado el test de Student para las variables continuas y la ChiCuadrado para las variables dicotómicas.

Tal como se muestra en la (Tabla 4), existen diversas variables con comportamientos estadísticamente significativos entre ambas sub-muestras. En este sentido, los equipos de trabajo de socias firmantes destinan menos horas a la realización de los trabajos, emiten por término medio menos opiniones con salvedades (OPINION) y sus clientes son peores en términos de rentabilidad, si se mide ésta en función de las pérdidas obtenidas en ejercicios anteriores (PYLOSS). Llama la atención que en términos porcentuales las socias auditoras no prestan servicios adicionales a los de auditoría (OTROSTRABAJOS). Este es un aspecto muy característico del segmento de las pequeñas firmas que, al contrario que las grandes operadoras del mercado, no aprovechan la cartera de clientes para incrementar sus ingresos con la prestación de otros servicios. Pues bien, esta característica se agudiza en el caso de que la auditora firmante sea socia de una pequeña o mediana firma. Finalmente, cabe destacar que las horas destinadas por término medio a la realización de trabajos resultan inferiores cuando el auditor firmante es mujer (HORAS), lo que se puede interpretar como

\begin{tabular}{|c|c|c|c|c|}
\hline \multicolumn{5}{|c|}{ Tabla 4. Comparación de medias en relación a la mediana de honorarios } \\
\hline Variable & $\begin{array}{l}\text { Observaciones } \\
\text { honorarios socio } \\
\text { auditor }\end{array}$ & $\begin{array}{c}\text { Observaciones } \\
\text { honorarios socia } \\
\text { auditora }\end{array}$ & Estadístico & Significatividad \\
\hline \multicolumn{5}{|c|}{ Panel A: variables continuas } \\
\hline LEV & 0,1222 & 0,1317 & $-0,917$ & 0,359 \\
\hline CATA & 1,0244 & 0,6334 & 0,296 & 0,767 \\
\hline ROA & 0,18 & $-0,001$ & 0,770 & 0,441 \\
\hline HORAS & 124,02 & 104,94 & 4,732 & 0,000 \\
\hline HONORARIOS & $6.182,61$ & $6.236,85$ & $-0,252$ & 0,801 \\
\hline \multicolumn{5}{|c|}{ Panel B: variables dicotómicas } \\
\hline PYLOSS & 0,07 & 0,16 & 19,007 & 0,00 \\
\hline OPINION & 0,24 & 0,12 & 17,000 & 0,00 \\
\hline NUEVO & 0,13 & 0,11 & 0,488 & 0,485 \\
\hline OTROSTRABAJOS & 0,12 & 0,00 & 26,360 & 0,00 \\
\hline CITY & 0,27 & 0,32 & 2,789 & 0,095 \\
\hline
\end{tabular}

Fuente: elaboración propia. 
una mejor eficiencia o como la preferencia de éstas por cubrir el riesgo a través de otras herramientas.

En la (Tabla 5) se recogen las correlaciones bivariadas entre todas las variables que se introducen en el modelo de precios a estimar. Muchas variables muestran estar correlacionadas entre sí, si bien los valores no resultanexcesivamentealtossiendosimilaresa losestudiosprevios realizados sobre la estimación de precios de auditoría. Al objeto de analizar si las correlaciones entre variables pueden afectar a los resultados de este estudio, se ha procedido a realizar el análisis del factor de inflación de la varianza, del cual se infiere que la multicolinealidad no resulta un problema en la estimación.

\subsection{Análisis multivariante}

En la (Tabla 6) se observa los coeficientes obtenidos de las variables experimentales. Ante la realización del modelo se ha tenido en cuenta los efectos fijos, el sector y el período analizado (2002-2014). El modelo resulta significativo, siendo la bondad de ajuste, según el coeficiente de determinación del modelo, de 87,10\%.

Del total de variables utilizadas para inferir el riesgo de negocio, tres de ellas han resultado estadísticamente significativas, si bien una de ellas con el signo contrario al esperado(NUEVO). La variable sexo, con la que se pretendía medir la relación entre la prima de riesgo y el género, resulta positiva y estadísticamente significativa. Se pone así de manifiesto que los honorarios son más altos para las mujeres que para los hombres que realizan el trabajo de auditoría en un 12,8\% aproximadamente. Dicho resultado es similar al alcanzado por Hardies et al. (2015), en el que aproximadamente los honorarios de las mujeres auditoras eran mayores en un $7 \%$ respecto a los honorarios de los hombres auditores.

\begin{tabular}{|c|c|c|c|c|c|c|c|c|c|c|}
\hline \multicolumn{11}{|c|}{ Tabla 5. Matriz correlaciones } \\
\hline & LEV & $V$ ROA & PYLOSS & CATA & OPINION & NUEVO & HORAS & $\begin{array}{c}\text { OTROS } \\
\text { TRABAJOS }\end{array}$ & SEX & CITY \\
\hline LEV & 1 &,- 035 &, $054^{* *}$ &,- 015 &, $042^{*}$ &,- 002 & ,180** & ,002 & ,018 &,$- 042^{*}$ \\
\hline $\mathrm{ROA}$ & & 1 &,- 020 & ,199** & -026 & ,022 &,- 014 & -014 &,- 015 & -021 \\
\hline PYLOSS & & & 1 & -006 & ,009 &,$- 043^{*}$ & ,031 & -010 &, $087^{* *}$ & 026 \\
\hline CATA & & & & 1 & -011 &,- 007 & ,003 & -009 & -006 & -012 \\
\hline OPINION & & & & & 1 & ,103* & $123^{* *}$ &,$- 058^{* *}$ &,$- 082^{* *}$ &, $052^{* *}$ \\
\hline NUEVO & & & & & & 1 & $-109^{* *}$ & $-042^{*}$ &,- 014 & -037 \\
\hline HORAS & & & & & & & 1 &, $061^{* *}$ & $-147^{* *}$ &, $041^{*}$ \\
\hline OTROSTRABAJOS & & & & & & & & 1 & $-102^{* *}$ &,$- 083^{*}$ \\
\hline SEX & & & & & & & & & 1 & 033 \\
\hline CITY & & & & & & & & & & 1 \\
\hline
\end{tabular}

La significación estadística viene expresada en asteriscos, a niveles superiores al 90\%(*), 95\%(**) y

99\%(***) de probabilidad

LEV: Deudas a largo plazo/Total de Activo

ROA: Beneficio de actividades ordinarias/Total de Activo

PYLOSS: 1 si la empresa tiene pérdidas en el año anterior y 0 en caso contrario

CATA: Activo circulante/total activo

OPINION: 1 si el informe de auditoría posee alguna salvedad y 0 cuando es favorable.

NUEVO: 1 cuando el cliente es nuevo y 0 en caso contrario

HORAS: Logaritmo neperiano de las horas declaradas por los auditores en cada encargo a la auditoría.

OTROSTRABJOS: Logaritmo neperiano de los ingresos percibidos por servicios distintos a la auditoría.

SEX: 1 si el auditor es mujer y 0 en el caso contrario

CITY: 1 si la firma tiene sede en Madrid o Barcelona y 0 en el caso contrario.

Fuente: elaboración propia. 
Tabla 6. Regresión modelo

\begin{tabular}{|c|c|c|c|}
\hline & $\begin{array}{c}\text { SIGNO } \\
\text { ESPERADO }\end{array}$ & COEFICIENTE & ESTADÍSTICO \\
\hline \multicolumn{4}{|c|}{ Variables experimentales } \\
\hline LEV & + & $-0,005$ & $-0,695$ \\
\hline ROA & - & 0,002 & 0,238 \\
\hline PYLOSS & + & 0,023 & $3,141^{\text {*** }}$ \\
\hline CATA & - & $-0,014$ & $-1,936 *$ \\
\hline OPINION & -- & 0,002 & 0,285 \\
\hline NUEVO & + & $-0,015$ & $-2,060^{* *}$ \\
\hline SEX & + & 0,128 & $16,857^{* * *}$ \\
\hline \multicolumn{4}{|c|}{ Variables de Control } \\
\hline HORAS & + & 0,929 & $118,242^{* * *}$ \\
\hline OTROSTRABAJOS & + & 0,026 & $3,472^{* * *}$ \\
\hline CITY & + & 0,028 & $3,741^{* * *}$ \\
\hline Sector & $\mathrm{Si}$ & & \\
\hline Temporal & $\mathrm{Si}$ & & \\
\hline \multicolumn{4}{|c|}{ Resumen del modelo } \\
\hline $\mathrm{R}^{2}$ & 0,871 & & \\
\hline \multicolumn{4}{|l|}{ Resumen ANOVA } \\
\hline EstadísticoF & $615,877^{* * *}$ & & \\
\hline
\end{tabular}

La significación estadística viene expresada en asteriscos, a niveles superiores al 90\%(*), 95\%(**) y 99\%(***) de probabilidad

LEV: Deudas a largo plazo/Total de Activo

ROA: Beneficio de actividades ordinarias/Total de Activo

PYLOSS: 1 si la empresa tiene pérdidas en el año anterior y 0 en caso contrario

CATA: Activo circulante/total activo

OPINION: 1 si el informe de auditoría posee alguna salvedad y 0 cuando es favorable.

NUEVO: 1 cuando el cliente es nuevo y 0 en caso contrario

HORAS: Logaritmo neperiano de las horas declaradas por los auditores en cada encargo a la auditoría. OTROSTRABJOS: Logaritmo neperiano de los ingresos percibidos por servicios distintos a la auditoría. SEX: 1 si el auditor es mujer y 0 en el caso contrario CITY: 1 si la firma tiene sede en Madrid o Barcelona y 0 en el caso contrario.

Fuente: elaboración propia.

Es decir, una vez tenidas en cuenta las variables contempladas por el auditor en su análisis del riesgo de negocio, las mujeres incrementan aún más esta prima, siendo el resultado similar a los estudios que aseguran que las mujeres tienen una adversidad superior al riesgo y mejores habilidades para la detección de éstos (Chung y Monroe, 2001; Gold et al. 2009; Ittonen y Peni, 2012). Luego se acepta la hipótesis de que las socias firmantes aplican una prima de riesgo superior que los auditores en los honorarios.

En cuanto a las variables con las que se pretendía capturar el riesgo de negocio a través del riesgo financiero del cliente (Tabla 6), la variable PYLOSS es estadísticamente significativa, siendo la relación esperada y la obtenida respecto a la variable HONORARIOS positiva. Por tanto, si la empresa auditada ha tenido pérdidas durante el ejercicio anterior, la firma auditora sí tendrá en cuenta dicha variable como un factor del riesgo de negocio, lo que supone la incorporación de una prima por riesgo en los precios del encargo. En consecuencia, si en el año anterior la empresa auditada presentó pérdidas, hay un incremento de aproximadamente un $2,3 \%$ en los precios.

La variable CATA es igualmente significativa y positiva. Ello implica quecuantomáslíquido sea el activo de la compañía, el auditor percibirá menor riesgo de negocio. Una mayor liquidez está relacionada con una mayor solvencia en el corto plazo, de tal forma que se reduce el riesgo de causar pérdidas a terceros por riesgo de impago, no dándose así la condición necesaria de que exista daño económico a terceros para que exista el riesgo de negocio. 
Por otro lado, en relación con las variables relacionadas con los factores del riesgo inherente, se obtiene que únicamente la variable NUEVO tiene una relación lineal significativa y con signo negativo. Contrario al signo esperado, pero similar al que obtuvieron Monterrey y Sánchez (2007), se pone de manifiesto que la competencia, vía precios, origina la necesidad de ampliar la cartera aplicando descuentos en los honorarios establecidos inicialmente. De esta forma, la reducción en el precio del servicio de un nuevo cliente es de aproximadamente un $1,5 \%$.

La variable de control HORAS es estadísticamente significativa respecto a la variable HONORARIOS y dicha relación es positiva; cuanto mayor sea el número de horas mayor será el precio del servicio (Bell et al., 2008).

En cuanto a la variable OTROS TRABAJOS, existe una relación positiva y significativa respecto a la variable dependiente, por lo que cuando se presten otros servicios distintos a la auditoría se produce un incremento del 2,6\% aproximadamente en los honorarios. Lejos de existir riesgo de independencia por el que el auditor reduce sus honorarios con el fin de conservar los obtenidos por ciertos servicios, los resultados reflejan la posibilidad de detección de riesgos adicionales durante la realización de otros trabajos que conducen al auditor al incremento de sus honorarios de auditoría. Por último, la variable CITY es estadísticamente significativa y además la relación esperada y la obtenida en el modelo es la misma. Esto pone de manifiesto, al igual que los resultados obtenidos por Chan et al. (1993), Brinn et al. (1994), O'Sullivan (2000), Beattie et al. (2001), que los honorarios se incrementan si la socia o socio firmante se encuentran en una gran ciudad (Barcelona o Madrid).

\subsection{Analisís de robustez}

Hasta el momento se ha estimado un modelo de honorarios, suponiendo que es estable ante cualquier característica de cada encargo. Tal y como se expone en la revisión de la literatura, la fijación de precios puede estar influida por otras características. Por ello, con el objetivo de comprobar que los resultados son robustos se plantea un análisis adicional utilizandootras especificaciones del modelo de estimación de precios, dividiendo la muestra en dos grupos. Se ha dividido el modelo de precios de auditoría en dos submuestras: la primera submuestra está formada por aquellos trabajos firmados por socias auditoras y la segunda por socios auditores, pretendiendo obtener que en ambas submuestras el modelo de prima de riesgos propuesto es válido.

Como se refleja en la (Tabla 7), el modelo de prima de riesgos es estadísticamente significativo para ambas submuestras, si bien la bondad de ajuste es inferior en el caso de la muestra de socias auditoras.

En primer lugar, si se tiene en cuenta la significatividad de las variables incluidas en el modelo para determinar la prima de riesgo, CATA y NUEVO resultan estadísticamente significativas en ambas submuestras, si bien la primera con signo distinto en función del género del auditor firmante. Para la submuestra de mujeres auditoras, la relación es negativa con respecto a los honorarios, esto es, perciben menor riesgo de negocio ante la existencia de un activo más líquido. Por el contrario, en la submuestra en la que los socios firmantes son varones la relación es positiva, conclusión similar a la que alcanzaron Gonthier-Besacier y Schatt (2007). Su origen puede residir en el riesgo inherente y de valoración subjetiva que contienen algunas partidas del activo corriente como pueden ser las existencias y los créditos comerciales, sobre los que el auditor tiene que pronunciarse en relación con su cuantía física (pudiendo existir distintas ubicaciones), su posible deterioro y su vencimiento.

Respecto a la variable NUEVO, se infiere que cuando el cliente es nuevo para la socia auditora se aplica un descuento en los precios del servicio de aproximadamente un 13,3\%, mientras que cuando lo es para un socio auditor dicho descuento es del 0,9\%. Como se menciona en el 
Tabla 7. Regresión modelo en submuestras

\begin{tabular}{|c|c|c|}
\hline VARIABLE & MUJER & HOMBRE \\
\hline & $\begin{array}{l}\text { COEFICIENTE } \\
\text { (ESTADISTICO) }\end{array}$ & $\begin{array}{l}\text { COEFICIENTE } \\
\text { (ESTADISTICO) }\end{array}$ \\
\hline \multicolumn{3}{|c|}{ Variables Experimentales } \\
\hline LEV & $\begin{array}{r}-0,130 \\
(-2,367)^{* *}\end{array}$ & $\begin{array}{r}-0,007 \\
(-1,274)\end{array}$ \\
\hline ROA & $\begin{array}{r}0,055 \\
(1,042)\end{array}$ & $\begin{array}{r}0,002 \\
(0,303)\end{array}$ \\
\hline PYLOSS & $\begin{array}{r}0,089 \\
(1,609) \\
\end{array}$ & $\begin{array}{r}0,006 \\
(1,168) \\
\end{array}$ \\
\hline CATA & $\begin{array}{r}0,232 \\
(4,150)^{* * *}\end{array}$ & $\begin{array}{r}-0,011 \\
(-1,842)^{*}\end{array}$ \\
\hline OPINION & $\begin{array}{r}0,085 \\
(1,571) \\
\end{array}$ & $\begin{array}{l}-0,002 \\
(0,378)\end{array}$ \\
\hline NUEVO & $\begin{array}{r}-0,133 \\
(-2,460)^{* *} \\
\end{array}$ & $\begin{array}{r}-0,009 \\
(-1,657)^{*} \\
\end{array}$ \\
\hline \multicolumn{3}{|c|}{ Variables de Control } \\
\hline HORAS & $\begin{array}{r}0,684 \\
(11,822)^{* * *}\end{array}$ & $\begin{array}{r}0,956 \\
(164,229)^{* * *}\end{array}$ \\
\hline OTROSTRABAJOS & $\begin{array}{r}0,066 \\
(1,149)\end{array}$ & $\begin{array}{r}0,026 \\
(4,549)^{* * *}\end{array}$ \\
\hline CITY & $\begin{array}{r}-0,175 \\
(-2,484)^{* *}\end{array}$ & $\begin{array}{r}0,053 \\
(9,243)^{* * *}\end{array}$ \\
\hline CONTROL SECTORIAL & SI & SI \\
\hline CONTROL TEMPORAL & SI & SI \\
\hline OBSERVACIONES & 207 & 2.329 \\
\hline $\mathbf{R}^{2}$ & 0,503 & 0,934 \\
\hline ESTADISTICO F & $10,640^{* * *}$ & $1.225,630^{* * *}$ \\
\hline
\end{tabular}

La significación estadística viene expresada en asteriscos, a niveles superiores al 90\%(*),

$95 \%{ }^{(*)}$ y $99 \%\left({ }^{* * *}\right)$ de probabilidad

LEV: Deudas a largo plazo/Total de Activo

ROA: Beneficio de actividades ordinarias/Total de Activo

PYLOSS: 1 si la empresa tiene pérdidas en el año anterior y 0 en caso contrario

CATA: Activo circulante/total activo

OPINION: 1 si el informe de auditoría posee alguna salvedad y 0 cuando es favorable.

NUEVO: 1 cuando el cliente es nuevo y 0 en caso contrario

HORAS: Logaritmo neperiano de las horas declaradas por los auditores en cada encargo.

OTROSTRABJ: Logaritmo neperiano de los ingresos percibidos por servicios distintos a la auditoría.

SEX: 1 si el auditor es mujer y 0 en el caso contrario

CITY: 1 si la firma de auditoría tiene sede en Madrid o Barcelona y 0 en el caso contrario.

Fuente: elaboración propia.

punto 4.2, este resultado pone de relieve que aunque el cliente presente mayor riesgo para la firma, la competencia existente en el mercado de auditoría español da lugar a la reducción de los precios para la captación de clientes.

Según los coeficientes obtenidos en las dos submuestras, la reducción de honorarios que realizan las socias firmantes es mayor que la de los socios firmantes, por lo que, a falta de un análisis más profundo, se podría evidenciar cierta dificultad en la captación de clientes por parte de la mujer que le obliga a reducir los honorarios en ese proceso de captación. 
Con respecto a las variables de control, y en línea con el párrafo anterior, la no existencia de una relación significativa entre la prestación de otros trabajos distintos a la auditoría y los honorarios en el caso de que sea mujer la socia firmante, venía anticipada en los análisis descriptivos. Y es que la prestación de otros servicios por socios firmantes es, en términos estadísticos, significativamente superior que en el caso de las mujeres, que podría considerarse casi nula. Se podría intuir que se trata de otra posible evidencia de diferencias de género a la hora de acceder a la prestación de servicios complementarios. Y es esa dificultad que ponen de manifiesto las variables NUEVO y OTROS TRABAJOS, la que vendría refrendada con la relación significativa y negativa de la variable CITY para el caso de que sean mujeres las firmantes. Si bien en el caso de los hombres, la prestación de servicios en grandes ciudades conlleva una prima, en el caso de las mujeres esa prima es negativa, es decir, para competir en los grandes mercados deben de disminuir sus precios con respecto a la competencia.

\section{Conclusiones}

El objetivo de este estudio ha sido investigar si las mujeres auditoras tienen en cuenta el riesgo de negocio que se puede presentar y si en la fijación de precios de cada uno de los contratos que mantienen con sus clientes tienen en cuenta los riesgos detectados en los mismos. Las socias firmantes incluyen una prima de riesgo para hacer frente a las posibles pérdidas futuras a las que se puede enfrentar, tales como la pérdida de reputación, litigios o sanciones. A diferencia de otros estudios relacionados, éste es el primero que estudia el impacto del género del socio firmante en las primas de riesgo aplicadas por las firmas españolas de pequeña y mediana dimensión.

La información utilizada en este estudio se ha obtenido de los datos comunicados al ICAC por las pequeñas y medianas firmas que han colaborado en la investigación, ya que según la Ley de Auditoría todas las firmas auditoras deberán comunicar en el modelo 03 determinada información relacionada con la actividad que realizan, así como el esfuerzo imputado a cada encargo.

En el tramo de mercado de pequeñas y medianas empresas en el que se centra este trabajo, el riesgo de negocio adquiere matices específicos. Tanto las firmas internacionales como las firmas participantes en este estudio deben soportar un nivel de riesgo de negocio. Sin embargo, al tratarse de un segmento de mercado en el que se compite vía precios existen ciertas dudas relativas a la posibilidad de aplicar primas sobre los precios como estrategia de cobertura. Tras utilizar un conjunto de variables para medir la situación financiera y el riesgo inherente a través de las cuales se infiere la posible existencia del riesgo de negocio, este estudio proporciona evidencia de que las socias firmantes tienen en cuenta aquellas variables que pueden afectar al riesgo de negocio; por ello, llevan a cabo la aplicación de una prima sobre los precios del servicio de auditoría.

Además, al tener en cuenta las horas se ha podido controlar la variable esfuerzo, descartando que dicho incremento de honorarios venga originado por la complejidad o por la propia cobertura del riesgo de auditoría.

Como resultados adicionales, utilizando dos submuestras en función del género, se concluye que mujeres y hombres auditores no reaccionan igual ante lo que podrían ser riesgos financieros e inherentes que originan el riesgo denegocio del auditor. Así, una mayor liquidez del activo es interpretada por las mujeres auditoras como una mejor solvencia y, por ende, un menor riesgo de negocio, mientras que los auditores hombres perciben un mayor riesgo, traducido éste en un incremento de honorarios, originado, tal vez, por el significativo grado de subjetividad que requiere la valoración de determinadas partidas del activo corriente.

Por último, si bien tanto hombres y mujeres utilizan el lowballing como estrategia de captación de clientes, las mujeres aplican descuentos de forma más agresiva, lo que 
se interpreta como una mayor dificultad para acceder al mercado, obligándola a reducir sus honorarios para captar clientes. Este hecho se ve refrendado en los encargos realizados en las grandes ciudades, ya que si bien los hombres repercuten unos honorarios mayores adaptándose al nivel de vida, las mujeres aplican descuentos, mostrando otra vez dificultades para prestar por primera vez servicios al cliente.

Este trabajo, sin embargo, no está exento de limitaciones y existe la posibilidad de que no se hayan controlado todos aquellos factores que pueden afectar al socio firmante, a los honorarios y a los factores determinantes de riesgo de control (Bell et al., 2001). En este sentido, variables como la experiencia del equipo anterior, el tamaño de la firma o el nivel general de endeudamiento del cliente podrían ser tenidos en cuenta en posteriores estudios.

En definitiva, analizando la aplicación de una prima de riesgo por parte de las mujeres auditoras se ha pretendido contribuir al debate sobre si existe diferencias de género a la hora de mostrar aversión, o no, al riesgo. Mientras que se ha puesto de manifiesto lo que podrían ser dificultades por parte de las socias firmantes en la captación de nuevos clientes, debiendo de aplicar honorarios aún más bajos que los hombres.

\section{Referencias}

Beattie, V., Goodacre, A., Pratt, K., \& Stevenson, J. (2001). The determinants of audit fees-evidence from the voluntary sector. Accounting and Business Research, 31(4), 243-274. https://doi.org/10.1080/00014 788.2001.9729619

Bell, T. B., Doogar, R., \& Solomon, I. (2008). Audit Labor Usage and Fees under Business Risk Auditing. Journal of Accounting Research, 46(4), 729-760. https://doi.org/10.1111/j.1475-679X.2008.00291.x

Bell, T. B., Landsman, W. R., \& Shackelford, D. A. (2001). Auditors' Perceived Business Risk and Audit Fees: Analysis and Evidence. Journal of Accounting Research, 39(1), 35-43. https://doi.org/10.1111/1475679X.00002

BOE. (2015). Ley 22/2015, de 20 de julio, de Auditoría de Cuentas. Retrieved from https://www.boe.es/eli/ es/1/2015/07/20/22

BOICAC. (2015). Norma internacional de auditoría de adaptación para su aplicación en España (NIA-ES) 200. Objetivos globales del auditor independiente y realización de la auditoría de conformidad con las normas internacionales de auditoría. Retrieved from http://www.icac.meh.es/

Brinn, T., Peel, M. J., \& Roberts, R. (1994). Audit Fee Determinants Of Independent \& Subsidiary Unquoted Companies In The Uk-An Exploratory Study. The British Accounting Review, 26(2), 101-121. https://doi. org/10.1006/bare.1994.1009

Byrnes, J. P., Miller, D. C., \& Schafer, W. D. (1999). Gender differences in risk taking: A meta-analysis. Psychological Bulletin, 125(3), 367-383. https://doi.org/10.1037/0033-2909.125.3.367

Carcello, J. V., \& Palmrose, Z.-V. (1994). Auditor Litigation and Modified Reporting on Bankrupt Clients. Journal of Accounting Research, 32, 1-30. https://doi.org/10.2307/2491436

Chan, P., Ezzamel, M., \& Gwilliam, D. (1993). Determinants of Audit Fees for Quoted UK Companies. Journal of Business Finance \& Accounting, 20(6), 765-786. https://doi.org/10.1111/j.1468-5957.1993.tb00292.x

Chung, J., \& Monroe, G. S. (2001). A Research Note on the Effects of Gender and Task Complexity on an Audit Judgment. Behavioral Research in Accounting, 13(1), 111-125. https://doi.org/10.2308/bria.2001.13.1.111

DeFond, M. L., Francis, J. R., \& Wong, T. J. (2000). Auditor Industry Specialization and Market Segmentation: Evidence from Hong Kong. AUDITING: A Journal of Practice \& Theory, 19(1), 49-66. https://doi.org/10.2308/aud.2000.19.1.49

Dwyer, P. D., Gilkeson, J. H., \& List, J. A. (2002). Gender differences in revealed risk taking: evidence from mutual fund investors. Economics Letters, 76(2), 151-158. https://doi.org/10.1016/S0165-1765(02)00045-9 
Francis, J. R., \& Simon, D. T. (1987). A Test of Audit Pricing in the Small-Client Segment of the U.S. Audit Market. The Accounting Review, 62(1), 145-157. Recovered www.jstor.org/stable/248051

Gold, A., Hunton, J. E., \& Gomaa, M. I. (2009). The Impact of Client and Auditor Gender on Auditors' Judgments (Retracted). Accounting Horizons, 23(1), 1-18. https://doi.org/10.2308/acch.2009.23.1.1

Gonthier-Besacier, N., \& Schatt, A. (2007). Determinants of audit fees for French quoted firms. Managerial Auditing Journal, 22(2), 139-160. https://doi.org/10.1108/02686900710718654

Graham, J. F., Stendardi, E. J., Myers, J. K., \& Graham, M. J. (2002). Gender differences in investment strategies: an information processing perspective. International Journal of Bank Marketing, 20(1), 17-26. https://doi.org/10.1108/02652320210415953

Hardies, K., Breesch, D., \& Branson, J. (2014). Do (Fe)Male Auditors Impair Audit Quality? Evidence from Modified Audit Opinions. SSRN Electronic Journal, 25(1). https://doi.org/10.2139/ssrn.1583206

Hardies, K., Breesch, D., \& Branson, J. (2015). The Female Audit Fee Premium. AUDITING: A Journal of Practice \& Theory, 34(4), 171-195. https://doi.org/10.2308/ajpt-51079

Houston, R. W., Peters, M. F., \& Pratt, J. H. (1999). The Audit Risk Model, Business Risk and Audit-Planning Decisions. The Accounting Review, 74(3), 281-298. https://doi.org/10.2308/accr.1999.74.3.281

Instituto de Censores Jurados de Cuentas de España (2016). Informe sobre la situación de la auditoría en España para los ejercicios 2006-2016. Madrid, España.

Ittonen, K. \& Vähämaa, E., \& Vähämaa, S. (2013). Female Auditors and Accruals Quality. Accounting Horizons, 27, 205-228. https://doi.org/10.2139/ssrn.2060797

Ittonen, K., \& Peni, E. (2012). Auditor's Gender and Audit Fees. International Journal of Auditing, 16(1), 1-18. https://doi.org/10.1111/j.1099-1123.2011.00438.x

Ittonen, K., Peni, E., \& Vähämaa, S. (2012). Female Auditors and Accruals Quality. SSRN Electronic Journal, 27(2), 205-228. https://doi.org/10.2139/ssrn.2060797

Jianakoplos, N. A., \& Bernasek, A. (1998). Are Women More Risk Averse? Economic Inquiry, 36(4), 620-630. https://doi.org/10.1111/j.1465-7295.1998.tb01740.x

Johnstone, K. M., \& Bedard, J. C. (2003). Risk Management in Client Acceptance Decisions. The Accounting Review, 78(4), 1003-1025. https://doi.org/10.2308/accr.2003.78.4.1003

Knechel, W. R., Niemi, L., \& Zerni, M. (2013). Empirical Evidence on the Implicit Determinants of Compensation in Big 4 Audit Partnerships. Journal of Accounting Research, 51(2), 349-387. https://doi. org/10.1111/1475-679X.12009

Lys, T., \& Watts, R. L. (1994). Lawsuits against Auditors. Journal of Accounting Research, 32, 65-93. https:// doi.org/10.2307/2491440

Monterrey-Mayoral, J., \& Sánchez-Segura, A. (2007). Un estudio empírico de los honorarios del auditor. Cuadernos de Economía y Dirección de La Empresa, 10(32), 81-109. https://doi.org/10.1016/S11385758(07)70092-0

Niemi, L. (2004). Auditor size and audit pricing: evidence from small audit firms. European Accounting Review, 13(3), 541-560. https://doi.org/10.1080/0963818042000237151

NORMA UNE-ISO 22301. (2015). ISO 22301: Gestión de la Continuidad del Negocio.

O'sullivan, N. (2000). The Impact of Board Composition and Ownership on Audit Quality: Evidence from Large UK Companies. The British Accounting Review, 32(4), 397-414. https://doi.org/10.1006/ bare.2000.0139

Olsen, R. A., \& Cox, C. M. (2001). The Influence of Gender on the Perception and Response to Investment Risk: The Case of Professional Investors. Journal of Psychology and Financial Markets, 2(1), 29-36. https://doi.org/10.1207/S15327760JPFM0201_3 
Palmrose, Z.-V. (1986). Audit Fees and Auditor Size: Further Evidence. Journal of Accounting Research, 24(1), 97. https://doi.org/10.2307/2490806

Pratt, J., \& Stice, J. D. (1994). The effects of client characteristics on auditor litigation risk judgments, required audit evidence, and recommended audit fees. The Accounting Review, 69(4), 639-656. Recovered www.jstor.org/stable/248435

Simunic, D. A. (1980). The Pricing of Audit Services: Theory and Evidence. Journal of Accounting Research, 18(1), 161. https://doi.org/10.2307/2490397

Simunic, D. A., \& Stein, M. T. (1996). The impact of litigation risk on audit pricing: A review of the economics and the evidence. Auditing: A Journal of Practice and Theory, 15, 119-134. https://www.researchgate. net/publication/246424855_The_Impact_of_Litigation_Risk_on_Audit_Pricing_A_Review_of_The_ Economics_and_the_Evidence

Stice, J. D. (1991). Using financial and market information to identify pre-engagement factors associated with lawsuits against auditors. The Accounting Review, 66(3), 516-533. https://www.jstor.org/ stable/247807

Sundén, A. E., \& Surette., B. J. (1998). Gender differences in the allocation of assets in retirement savings plans. The American Economic Review, 88(2), 207-211. https://www.jstor.org/stable/116920

Watson, J., \& McNaughton, M. (2007). Gender Differences in Risk Aversion and Expected Retirement Benefits. Financial Analysts Journal, 63(4), 52-62. https://doi.org/10.2469/faj.v63.n4.4749

Watson, J., \& Robinson, S. (2003). Adjusting for risk in comparing the performances of male- and female-controlled SMEs. Journal of Business Venturing, 18(6), 773-788. https://doi.org/10.1016/S08839026(02)00128-3

Zhan-Shu, S. (2000). Auditor resignations: clientele effects and legal liability. Journal of Accounting and Economics, 29(2), 173-205. https://doi.org/10.1016/S0165-4101(00)00019-7 